\title{
Comparison of risk assessment strategies for not-high-risk pulmonary embolism
}

\author{
Lukas Hobohm ${ }^{1,2}$, Kristian Hellenkamp ${ }^{3}$, Gerd Hasenfuß ${ }^{3,4}$, Thomas Münzel ${ }^{2,5,6}$, \\ Stavros Konstantinides ${ }^{1}$ and Mareike Lankeit ${ }^{1,3}$
}

\begin{abstract}
Affiliations: ${ }^{1}$ Center for Thrombosis and Hemostasis, University Medical Center of the Johannes Gutenberg University, Mainz, Germany. ${ }^{2}$ Center for Cardiology, Cardiology I, University Medical Center of the Johannes Gutenberg University, Mainz, Germany. ${ }^{3}$ Clinic of Cardiology and Pneumology, Heart Center, University of Göttingen, Göttingen, Germany. ${ }^{4}$ German Center for Cardiovascular Research, Partner Site Göttingen, Göttingen, Germany. ${ }^{5}$ German Center for Cardiovascular Research, Partner Site Rhein-Main, Mainz, Germany. ${ }^{6}$ Center for Translational Vascular Biology, University Medical Center of the Johannes Gutenberg University, Mainz, Germany.
\end{abstract}

Correspondence: Mareike Lankeit, Center for Thrombosis and Hemostasis, University Medical Center Mainz, Johannes Gutenberg University of Mainz, Langenbeckstraße 1, 55131 Mainz, Germany.

E-mail: mlankeitवuni-mainz.de

ABSTRACT We compared the prognostic performance of the 2014 European Society of Cardiology (ESC) risk stratification algorithm with the previous 2008 ESC algorithm, the Bova score and the modified FAST score (based on a positive heart-type fatty acid-binding protein (H-FABP) test, syncope and tachycardia, modified using high-sensitivity troponin $\mathrm{T}$ instead of $\mathrm{H}-\mathrm{FABP}$ ) in 388 normotensive pulmonary embolism patients included in a single-centre cohort study.

Overall, 25 patients (6.4\%) had an adverse 30-day outcome. Regardless of the score or algorithm used, the rate of an adverse outcome was highest in the intermediate-high-risk classes, while all patients classified as low-risk had a favourable outcome (no pulmonary embolism-related deaths, 0-1.4\% adverse outcome). The area under the curve for predicting an adverse outcome was higher for the 2014 ESC algorithm $(0.76,95 \%$ CI $0.68-0.84)$ compared with the 2008 ESC algorithm (0.65, 95\% CI 0.56-0.73) and highest for the modified FAST score $(0.82,95 \%$ CI $0.75-0.89)$. Patients classified as intermediate-high-risk by the 2014 ESC algorithm had a 8.9-fold increased risk for an adverse outcome (3.2-24.2, $\mathrm{p}<0.001$ compared with intermediate-low- and low-risk patients), while the highest OR was observed for a modified FAST score $\geqslant 3$ points (OR 15.9, 95\% CI 5.3-47.6, $\mathrm{p}<0.001$ ).

The 2014 ESC algorithm improves risk stratification of not-high-risk pulmonary embolism compared with the 2008 ESC algorithm. All scores and algorithms accurately identified low-risk patients, while the modified FAST score appears more suitable to identify intermediate-high-risk patients.

@ERSpublications

2014 ESC guideline algorithm improves risk stratification of not-high-risk PE compared with 2008 ESC algorithm http://ow.ly/UT0K6

For editorial comment see Eur Respir J 2016; 47: 1054-1056 [DOI: 10.1183/13993003.02007-2015].

This article has supplementary material available from erj.ersjournals.com

Received: Sept 262015 | Accepted after revision: Nov 122015 | First published online: Jan 072016

Support statement: This study was supported by the German Federal Ministry of Education and Research (BMBF $01 \mathrm{EO} 1003$ and BMBF 01EO1503). The authors are responsible for the contents of this publication. Funding information for this article has been deposited with FundRef.

Conflict of interest: Disclosures can be found alongside the online version of this article at erj.ersjournals.com

Copyright OERS 2016 


\section{Introduction}

Risk stratification is mandatory to guide risk-adjusted management of patients with acute pulmonary embolism (PE) [1]. Therapeutic implications according to the patients' risk strata were demonstrated by recent randomised trials. While patients with a low risk of an early adverse outcome or death may be candidates for early discharge and continuation of treatment at home, if proper outpatient care and anticoagulant treatment can be provided [1-4], early reperfusion treatment (e.g. systemic thrombolysis) should be considered for patients with intermediate-high-risk PE and clinical signs of haemodynamic decompensation $[1,5,6]$. Based on numerous scores and models for risk stratification of not-high-risk PE developed and validated during the past years, a new algorithm for risk assessment was introduced by the 2014 European Society of Cardiology (ESC) guidelines. Compared with the 2008 ESC guidelines, the most comprehensive innovation was the introduction of the (simplified) Pulmonary Embolism Severity Index ((s)PESI [7]; table 1) as the first step of the risk assessment algorithm of not-high-risk PE [1]. However, the proposed stepwise algorithm has never been tested in a controlled management trial.

Additionally, combination models such as the FAST score (based on a positive heart-type fatty acid-binding protein (H-FABP) test, syncope and tachycardia) $[8,9]$ and the Bova score $[10,11]$ (table 1) were developed and validated, with the aim to identify not-high-risk PE patients with an intermediate-high risk of early complications. Since the FAST score requires measurement of H-FABP, a biomarker of myocardial injury, which is not routinely available in the majority of hospitals, we tested in the present study whether replacement of H-FABP by high-sensitivity troponin $\mathrm{T}$ (hsTnT) provides comparable prognostic information. Additionally, we compared the performance of the new algorithm proposed by the 2014 ESC guidelines with the algorithm proposed by the 2008 ESC guidelines [12] and the modified FAST score and the Bova score for risk stratification of not-high-risk PE.

\section{Material and methods}

\section{Patient cohort and study design}

Consecutive patients aged $\geqslant 18$ years with confirmed acute PE were included in a single-centre prospective cohort study (Pulmonary Embolism Registry of Göttingen (PERGO)) at the University Medical Center Göttingen, Germany between September 2007 and August 2014. The study protocol has been described in detail previously $[9,13]$. As shown in figure 1, patients were excluded from analysis if they fulfilled one of the following criteria: 1) withdrawal of written consent for participation in the study, 2) missing hsTnT plasma concentrations on admission, 3) haemodynamic instability (defined as systolic blood pressure $<90 \mathrm{mmHg}$ or a pressure drop of $>40 \mathrm{mmHg}$ for $15 \mathrm{~min}$ at presentation), 4) unexpected or accidental diagnosis of PE (patients undergoing diagnostic tests for another suspected disease), 5) acute left heart failure or acute respiratory failure responsible for symptoms, 6) missing data or 7) recurrent PE (only the first event was included in the analysis).

Local clinical pathways strongly recommended a transthoracic echocardiogram (TTE) within $48 \mathrm{~h}$ of admission. Right ventricular (RV) dysfunction on TTE was defined as RV dilatation combined with absence

\begin{tabular}{|c|c|c|c|}
\hline & sPESI [7] & Bova score $[10,11]$ & FAST score $[8,9]$ \\
\hline \multirow[t]{3}{*}{ Items (points) } & Cancer (1) & $\begin{array}{c}\text { Elevated cardiac } \\
\text { troponin }^{\#}(2)\end{array}$ & $\mathrm{H}-\mathrm{FABP} \geqslant 6 \mathrm{ng} \cdot \mathrm{mL}^{-1 \pi}(1.5)$ \\
\hline & $\begin{array}{c}\text { Cardiopulmonary } \\
\text { disease (1) }\end{array}$ & $\begin{array}{l}\text { RV dysfunction } \\
\text { (TTE or CT) (2) }\end{array}$ & Syncope (1.5) \\
\hline & $\begin{array}{c}\mathrm{HR} \geqslant 110 \text { beats } \cdot \mathrm{min}^{-1}(1) \\
\text { sBP } 90-100 \mathrm{mmHg}(1) \\
\mathrm{SaO}_{2}<90 \%(1) \\
\text { Age }>80 \text { years }(1)\end{array}$ & $\begin{array}{l}\mathrm{HR} \geqslant 110 \text { beats } \cdot \mathrm{min}^{-1}(1) \\
\mathrm{sBP} 90-100 \mathrm{mmHg}(2)\end{array}$ & $H R \geqslant 100$ beats $\cdot \min ^{-1}(2)$ \\
\hline \multicolumn{4}{|l|}{ Risk class } \\
\hline Low-risk & 0 points & $0-2$ points & $<3$ points \\
\hline Intermediate-low-risk & & 3-4 points & \\
\hline Intermediate-high-risk & $\geqslant 1$ point & $>4$ points & $\geqslant 3$ points \\
\hline
\end{tabular}

sPESI: simplified Pulmonary Embolism Severity Index; FAST score: based on a positive heart-type fatty acid-binding protein (H-FABP) test, syncope and tachycardia; RV: right ventricular; TTE: transthoracic echocardiography; CT: computed tomography; HR: heart rate; SBP: systolic blood pressure; $\mathrm{SaO}_{2}$ : arterial oxygen saturation. \#: high-sensitivity troponin $\mathrm{T}(\mathrm{hSTnT}) \geqslant 14 \mathrm{pg} \cdot \mathrm{mL}^{-1}$ for patients $<75$ years and $\geqslant 45 \mathrm{pg} \cdot \mathrm{mL}^{-1}$ for patients $\geqslant 75$ years; 1 : replaced by $\mathrm{hsTnT} \geqslant 14 \mathrm{pg} \cdot \mathrm{mL}^{-1}$ for patients $<75$ years and $\geqslant 45 \mathrm{pg} \cdot \mathrm{mL}^{-1}$ for patients $\geqslant 75$ years. 
525 patients $\geqslant 18$ years with objectively confirmed PE included in PERGO between September 2007 and August 2014

Exclusion criteria:

90 patients without measurement of hsTnT plasma concentration on admission

32 patients with haemodynamic instability

6 patients with diagnosis of PE as an accidental/asymptomatic finding during work-up for another suspected disease

7 patients with acute left heart failure or acute respiratory failure responsible for symptoms

1 patient with missing data

1 patient with recurrent PE (first event was included in the analysis)

388 patients included in the analysis

FIGURE 1 Flow diagram of exclusion criteria. PE: pulmonary embolism; PERGO: Pulmonary Embolism Registry Göttingen; hsTnT: high-sensitivity troponin T.

of the inspiratory collapse of the inferior vena cava or an elevated systolic gradient through the tricuspid valve $(>30 \mathrm{mmHg})$, in the absence of left ventricular $(\mathrm{LV})$ or mitral valve disease. RV dysfunction on (diagnostic) computed tomography (CT) was assessed by an experienced radiologist based on the following criteria: 1) RV dilatation (RV/LV ratio $\geqslant 1.0), 2$ ) right atrial dilatation, 3) dilatation of the pulmonary arteries and 4) contrast reflux in the inferior vena cava. RV dysfunction was defined as RV dilatation (RV/LV ratio $\geqslant 1.0$ ) or presence of at least two of the latter three findings.

Patients were stratified in risk classes according to the algorithms proposed by the 2008 ESC guidelines [12] and the 2014 ESC guidelines [1], and by the modified FAST score [8,9] (table 1) and the Bova score $[10,11]$ (table 1). The sPESI [7] (table 1) was used for stratification in risk classes according to the 2014 ESC algorithm. For calculation of the modified FAST score, "H-FABP $\geqslant 6 \mathrm{ng} \cdot \mathrm{mL}^{-1}$ " was replaced by an age-adjusted hsTnT cut-off value $\left(\geqslant 14 \mathrm{pg} \cdot \mathrm{mL}^{-1}\right.$ for patients $<75$ years and $\geqslant 45 \mathrm{pg} \cdot \mathrm{mL}^{-1}$ for patients $\geqslant 75$ years, as previously reported [14]) determined at the time of admission. This hsTnT cut-off value was also used for classification in risk classes using the 2008 and 2014 ESC algorithms and the Bova score. For calculation of all algorithms and scores, missing values were considered to be normal [15]. Elevated $\mathrm{N}$-terminal pro-brain natriuretic peptide plasma concentrations were defined as levels $\geqslant 600 \mathrm{pg} \cdot \mathrm{mL}^{-1}$, as previously reported [16].

All patients were followed over a period of 30 days. The primary outcome was an adverse 30-day outcome defined as PE-related death, need for mechanical ventilation, cardiopulmonary resuscitation or the administration of catecholamines (except for dopamine at an infusion rate of $\leqslant 5 \mu \mathrm{g} \cdot \mathrm{kg}$ body weight $^{-1} \cdot \mathrm{min}^{-1}$ ). The secondary outcome was 30 -day all-cause mortality. Three of the authors (L. Hobohm, K. Hellenkamp and M. Lankeit) independently adjudicated causes of death by reviewing the medical records and autopsy reports (if performed). Death was determined to be PE-related if it was either confirmed by autopsy, following a clinically severe episode of acute PE or in the absence of an alternative diagnosis.

Treatment decisions were made by the physicians caring for the patient according to current guidelines [12] and were not influenced by the study protocol. Study results were not communicated to the clinicians and thus not used to guide patient management, or to monitor effects of treatment during the hospital stay or at any time during the 30-day follow-up period. The study protocol was conducted in accordance with the amended Declaration of Helsinki and was approved by the local independent Ethic Committee of the Medical University Göttingen, Germany; all patients gave informed written consent for participation in the study.

\section{Statistical analysis}

Fisher's exact test or Chi-squared test was used to compare categorical variables, which are expressed as absolute number or percentage. Continuous variables were found not to follow a normal distribution when tested with the modified Kolmogorov-Smirnov test (Lilliefors test); therefore, these variables are expressed as medians with the corresponding interquartile range and were compared using the unpaired Mann-Whitney U-test. Receiver operating characteristic (ROC) curve analysis was performed to determine the area under the curve (AUC) of algorithms and scores (absolute points) with regard to study outcomes. To allow comparison of algorithms and scores, the three-level 2014 ESC algorithm and Bova score were 
dichotomised as low- and intermediate-low-risk ("low-risk") versus intermediate-high-risk ("intermediate-high-risk") [11]. The McNemar-Bowker test was used to compare the distribution of patients by the classification of different dichotomous risk assessment strategies. Comparison of the prognostic performance of dichotomous algorithms and scores was performed by calculation of sensitivity, specificity, positive predictive value (PPV) and negative predictive value (NPV), and positive and negative likelihood ratios. The prognostic relevance of dichotomous algorithms and scores as well as single predictors with regard to study outcomes was tested using univariable logistic regression analysis and presented as odds ratios with the corresponding 95\% confidence intervals. Additionally, each algorithm and score was tested separately in a multivariable logistic regression model also including each single univariable predictor identified by univariable analysis.

A two-sided significance level of $\alpha<0.05$ was defined appropriate to indicate statistical significance. Statistical analyses were performed using SPSS version 21.0 (SPSS, Chicago, IL, USA).

\section{Results}

Baseline findings and clinical outcomes

Between September 2007 and August 2014, 525 patients $\geqslant 18$ years with objectively confirmed PE were included in PERGO and 388 patients (73.9\%) were included in the analysis (figure 1). The baseline characteristics of the study patients are shown in table 2 . hsTnT plasma concentrations were measured in all patients on admission and were elevated $\left(\geqslant 14 \mathrm{pg} \cdot \mathrm{mL}^{-1}\right.$ for patients $<75$ years and $\geqslant 45 \mathrm{pg} \cdot \mathrm{mL}^{-1}$ for patients $\geqslant 75$ years [14]) in 194 patients $(50.0 \%)$. In 379 patients (97.7\%), RV (dys-)function was assessed on CT and/or TTE: TTE was performed in 239 patients (61.6\%), with evidence of RV dysfunction in 118 (49.4\%). In 360 patients (92.8\%), RV function could be assessed on (diagnostic) CT; of those, 175 (48.7\%) were diagnosed with RV dysfunction.

TABLE 2 Baseline characteristics, medical history and initial presentation of 388 normotensive patients with acute pulmonary embolism (PE)

\begin{tabular}{|c|c|c|c|c|}
\hline & All study patients & Modified FAST score $\geqslant 3$ points & Modified FAST score $<3$ points & p-value \\
\hline Male & $179(46.1)$ & $44(39.6)$ & $135(48.7)$ & 0.104 \\
\hline \multicolumn{5}{|l|}{ Risk factors for VTE and comorbidities } \\
\hline Previous VTE & $122(31.4)$ & 25 (22.5) & 97 (35.0) & 0.021 \\
\hline Immobilisation/trauma/surgery ${ }^{\#}$ & $121(31.2)$ & $31(27.9)$ & 90 (32.5) & 0.399 \\
\hline Unprovoked $P E^{-1}$ & $216 / 387(55.8)$ & $64(57.7)$ & $152 / 276(55.1)$ & 0.653 \\
\hline Chronic heart failure & 64 (16.5) & $18(16.2)$ & $46(16.6)$ & 1.000 \\
\hline Chronic pulmonary disease & $53(13.7)$ & $17(15.3)$ & $36(13.0)$ & 0.624 \\
\hline Renal insufficiency $\S$ & $118(30.4)$ & $38(34.2)$ & $80(28.9)$ & 0.329 \\
\hline \multicolumn{5}{|l|}{$\begin{array}{l}\text { Symptoms and clinical findings } \\
\text { on admission }\end{array}$} \\
\hline Symptom duration $<24 \mathrm{~h}$ & 195 (50.3) & $73(65.8)$ & $122(44.0)$ & $<0.001$ \\
\hline Chest pain & $221 / 387(57.1)$ & $45(40.5)$ & $176 / 276(63.8)$ & $<0.001$ \\
\hline Hypoxia"\# & $89 / 331(26.9)$ & $43 / 101(42.6)$ & $46 / 230(20.0)$ & $<0.001$ \\
\hline sPESI $\geqslant 1$ point & $254(65.5)$ & $96(86.5)$ & $158(57.0)$ & $<0.001$ \\
\hline RV dysfunction on TTE and/or CT & $202 / 379(52.3)$ & $90 / 110(81.8)$ & $112 / 269(41.6)$ & $<0.001$ \\
\hline hsTnT $\geqslant$ age-adjusted cut-off value & $194(50.0)$ & $106(95.5)$ & $88(31.8)$ & $<0.001$ \\
\hline $\mathrm{NT}-$ proBNP $\geqslant 600 \mathrm{pg} \cdot \mathrm{mL}^{-1}$ & $191 / 362(52.8)$ & $76 / 102(74.5)$ & $115 / 260(44.2)$ & $<0.001$ \\
\hline \multicolumn{5}{|c|}{$\begin{array}{l}\text { Data are presented as } \mathrm{n}, \mathrm{n}(\%) \text { or median (interquartile range), unless otherwise stated. Patients were stratified according the modified FAST } \\
\text { score (see table } 1 \text { for definition). VTE: venous thromboembolism; sPESI: simplified Pulmonary Embolism Severity Index; RV: right ventricular; } \\
\text { TTE: transthoracic echocardiography; CT: computed tomography; hsTnT: high-sensitivity troponin T; NT-proBNP: N-terminal pro-brain natriuretic } \\
\text { peptide. \#: within the past } 4 \text { weeks; }{ }^{\uparrow}: \text { a PE in absence of a temporary or reversible risk factor such as surgery, trauma, immobilisation, } \\
\text { contraceptives or hormone replacement, pregnancy; }{ }^{+}: \text {active or antitumour therapy within the last } 6 \text { months, or metastatic state; }{ }^{\S} \text { : glomerular } \\
\text { filtration rate }<60 \mathrm{~mL} \cdot \mathrm{min}^{-1} \cdot 1.73 \mathrm{~m}^{-2} \text { body surface area; }{ }^{f}: \text { systolic blood pressure } 90-100 \mathrm{mmHg} \text { on admission; } \# \# \text { : arterial oxygen saturation } \\
<90 \% \text { or arterial oxygen partial pressure }<60 \mathrm{mmHg} ;{ }^{\uparrow 1}: \geqslant 14 \mathrm{pg} \cdot \mathrm{mL}^{-1} \text { for patients }<75 \text { years and } \geqslant 45 \mathrm{pg} \cdot \mathrm{mL}^{-1} \text { for patients } \geqslant 75 \text { years. }\end{array}$} \\
\hline
\end{tabular}


A total of 53 patients (13.7\%) received reperfusion therapy; of those, 18 patients received early systemic thrombolysis (within $24 \mathrm{~h}$ ), 34 patients were included in the "Pulmonary Embolism Thrombolysis" (PEITHO) study [6] and randomised to either single-bolus tenecteplase or placebo, and in four patients surgical embolectomy was performed. On discharge, 54 patients (13.9\%) were treated with low-molecular-weight heparin or fondaparinux (e.g. in case of active malignancy or international normalised ratio <2.0), $264(68.0 \%)$ with vitamin $K$ antagonist and 43 (11.1\%) with non-vitamin-K-dependent novel oral anticoagulants (predominantly rivaroxaban).

During the first 30 days, 25 patients (6.4\%) had an adverse outcome and 14 (3.6\%) died. Death was adjudicated to be due to $\mathrm{PE}$ in eight patients $(2.1 \%)$.

\section{Performance of the modified FAST score}

As shown in table 2, 111 patients $(28.6 \%)$ were classified in the intermediate-high-risk class ( $\geqslant 3$ points) using the modified FAST score. Compared with patients classified as low-risk (modified FAST score $<3$ points; table 2), these patients presented more often with symptoms and clinical findings indicating more severe PE and more often reached the primary outcome (adverse 30-day outcome $1.4 \%$ versus $18.9 \%$, $\mathrm{p}<0.001$ ) and secondary outcome (all-cause mortality $0.7 \%$ versus $10.8 \%, \mathrm{p}<0.001$; PE-related mortality $0 \%$ versus $7.2 \%, \mathrm{p}<0.001$ ). A modified FAST score $\geqslant 3$ points was associated with a 15.9 -fold increased risk to reach the primary outcome ( $95 \%$ CI 5.3-47.6, $\mathrm{p}<0.001$; table 3 ) and a 16.7 -fold increased risk to reach the secondary outcome (95\% CI 3.7-75.8, p<0.001).

\section{Comparison of algorithms and scores}

As described in the Material and methods section, patients were stratified to risk classes using the algorithms proposed by the 2008 [12] and 2014 [1] ESC guidelines, and the Bova score [10, 11] and the modified FAST score [8, 9]. As shown in figure 2a, the 2008 and 2014 ESC algorithm classified a larger number of patients in the intermediate-(high-) risk classes, while the Bova and the modified FAST score classified more patients in the low-risk classes. While none of the patients with a modified FAST score $\geqslant 3$ points was classified as low-risk by the 2014 ESC algorithm, $20.9 \%$ of the patients with a modified FAST score $<3$ points were stratified to the intermediate-high-risk category by the use of the 2014 ESC algorithm; of those 58 patients, three (5.2\%) had an adverse 30-day outcome. Similar results were observed for the Bova score in comparison with the 2014 ESC algorithm: good agreement was reached in classification of low-risk patients, while 13 patients classified as low-risk by the Bova score (6.2\%) were stratified to the intermediate-high-risk group by the 2014 ESC algorithm. Comparing the dichotomised algorithm proposed by the 2014 ESC guidelines with that of the 2008 ESC guidelines, more patients were categorised as low-risk $(65.7 \%$ versus $26.8 \%, \mathrm{p}<0.001)$ and more patients were classified as intermediate-high-risk by the modified FAST score compared with the dichotomised Bova score $(28.6 \%$ versus $16.2 \%, \mathrm{p}<0.001)$.

Importantly, regardless of the score or algorithm used, the risk of an adverse 30-day outcome was highest in the intermediate-high-risk classes, while all patients classified in low-risk classes had a favourable 30 -day outcome (no PE-related death, rate of an adverse outcome 0-1.4\%; figure 2b). Conformably, in all scores and algorithms, an increase in risk class was associated with a higher incidence of an adverse 30-day outcome. ROC analysis revealed a larger AUC with regard to an adverse 30-day outcome for the 2014 ESC algorithm (AUC 0.76) compared with the 2008 ESC algorithm (AUC 0.65), and the largest AUCs for the Bova score and modified FAST score (AUC 0.80 and 0.82, respectively; table 3 and figure 3).

TABLE 3 Prognostic performance of risk assessment strategies with regard to an adverse 30 -day outcome

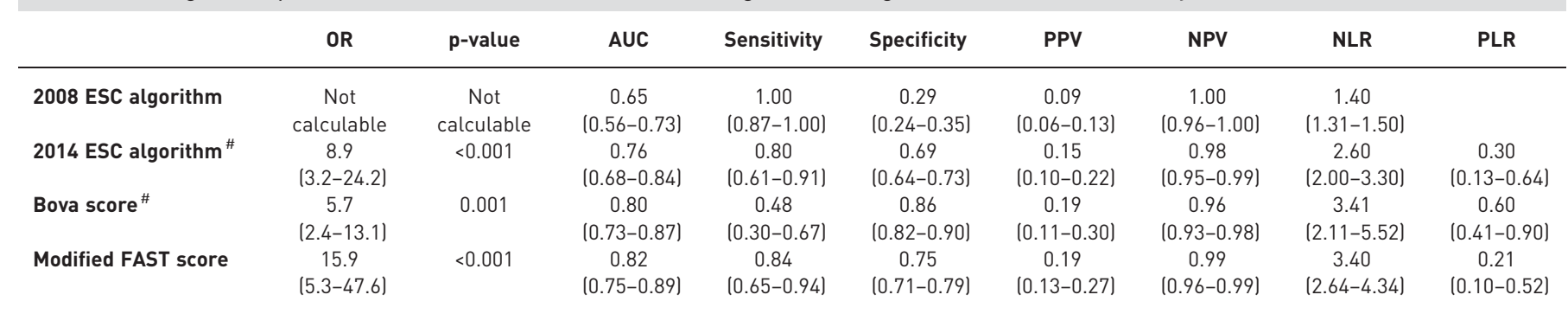

Data are presented as n (95\% CI), unless otherwise stated. AUC: area under the curve; PPV: positive predictive value; NPV: negative predictive value; NLR: negative likelihood ratio; PLR: positive likelihood ratio; ESC: European Society of Cardiology. \#: the three-level 2014 ESC algorithm and Bova score were dichotomised as described in the Material and methods section. The modified FAST score is defined in table 1. 

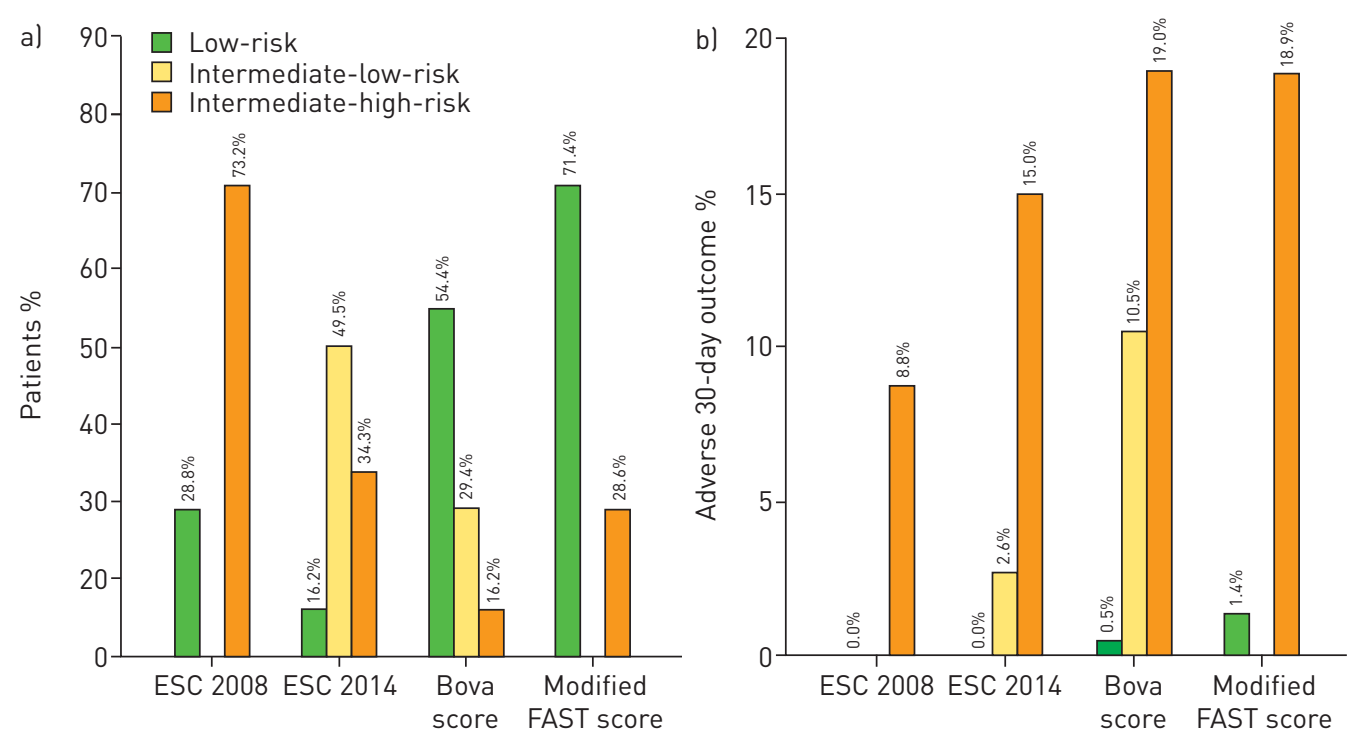

FIGURE 2 Performance of algorithms and score for risk assessment of acute pulmonary embolism. a) Classification in low-, intermediate-low- and intermediate-high-risk classes. b) Rate of an adverse 30-day outcome. While the 2014 European Society of Cardiology (ESC) algorithm and the Bova score stratify patients in three risk classes, the 2008 ESC algorithm and the modified FAST score (see table 1 for definition) stratify patients in two risk classes.

As described in the Material and methods section, the three-level 2014 ESC algorithm and Bova score were dichotomised to allow for comparison of the prognostic performance. The 2008 ESC algorithm was associated with an excellent NPV, while the PPV was highest for the Bova score and modified FAST score (table 3).

Patients classified as intermediate-high-risk by the 2014 ESC algorithm had an 8.9-fold increased risk for an adverse 30-day outcome (table 3). The highest OR was observed for patients classified as intermediate-high-risk by the modified FAST score. Additionally, as shown in table 4, a number of variables were identified as predictors of an adverse 30-day outcome using univariable logistic regression analysis. Of note, if included in a multivariable model (as described in the Material and methods section), all algorithms and scores remained independent predictors of an adverse 30-day outcome.

While the modified FAST score (OR 16.7, 95\% CI 3.7-75.8, p<0.001) and the 2014 ESC algorithm (OR 5.1, 95\% CI 1.6-16.6, $\mathrm{p}=0.007$ ) were able to predict all-cause 30-day mortality, the $2008 \mathrm{ESC}$ algorithm (OR 4.9, 95\% CI 0.6-38.2, $\mathrm{p}=0.126$ ) and the Bova score (OR 2.1, 95\% CI 0.6-7.0, $\mathrm{p}=0.212$ ) did not provide prognostic information.

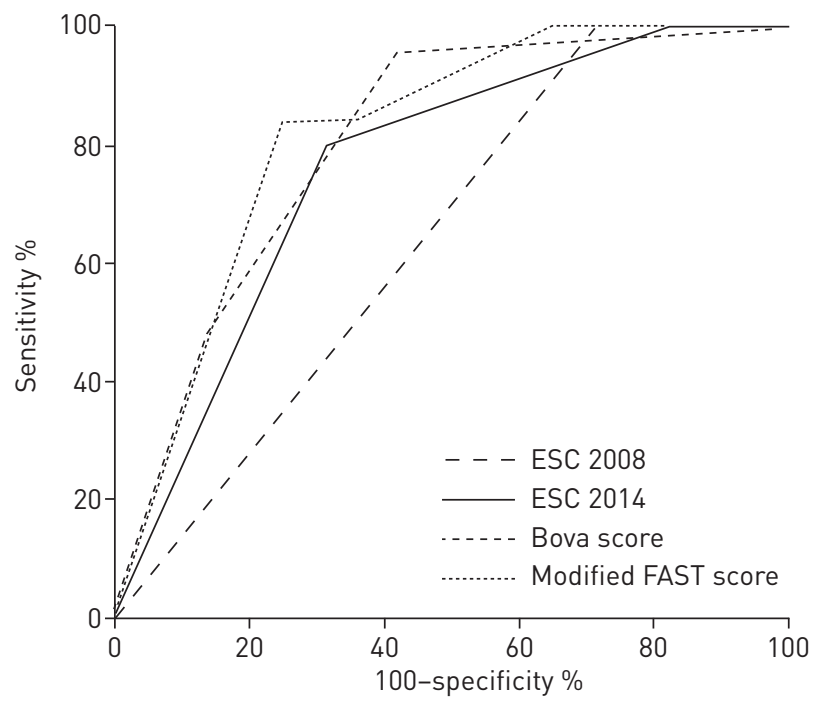

FIGURE 3 Receiver operating characteristic analysis of risk assessment strategies with regard to an adverse 30-day outcome. 


\begin{tabular}{|c|c|c|}
\hline & OR $(95 \% \mathrm{CI})$ & p-value \\
\hline \multicolumn{3}{|l|}{ Comorbidities } \\
\hline Chronic heart failure & $3.8(1.6-8.9)$ & 0.002 \\
\hline Renal insufficiency & $3.2(1.4-7.2)$ & 0.006 \\
\hline \multicolumn{3}{|c|}{ Symptoms and clinical findings on admission } \\
\hline Syncope & $2.9(1.1-7.3)$ & 0.025 \\
\hline Tachycardia $\geqslant 100$ beats $\cdot \mathrm{min}^{-1}$ & $5.5(2.2-13.5)$ & $<0.001$ \\
\hline sPESI $\geqslant 1$ point & $13.9(1.9-103.8)$ & 0.010 \\
\hline RV dysfunction on TTE and/or CT & $5.0(1.7-14.9)$ & 0.004 \\
\hline hsTnT $\geqslant$ age-adjusted cut-off value $\#$ & $27.3(3.6-203.6)$ & 0.001 \\
\hline NT-proBNP $\geqslant 600 \mathrm{pg} \cdot \mathrm{mL}^{-1}$ & $13.3(3.1-57.0)$ & 0.001 \\
\hline
\end{tabular}

SPESI: simplified Pulmonary Embolism Severity Index; RV: right ventricular; TTE: transthoracic echocardiography; CT: computed tomography; hsTnT: high-sensitivity troponin T; NT-proBNP: N-terminal pro-brain natriuretic peptide. ${ }^{\#}: \geqslant 14 \mathrm{pg} \cdot \mathrm{mL}^{-1}$ for patients $<75$ years and $\geqslant 45 \mathrm{pg} \cdot \mathrm{mL}^{-1}$ for patients $\geqslant 75$ years.

\section{Discussion}

Recently, the therapeutic implications of risk stratification of normotensive (not-high-risk) PE patients have been demonstrated in randomised trials. Evidence is accumulating that patients with low-risk PE should be considered for early discharge and continuation of treatment at home if proper outpatient care and anticoagulant treatment can be provided [1]. For example, in the "Outpatient versus inpatient Treatment for patients with acute Pulmonary Embolism" (OTPE) study, for those patients selected for outpatient treatment based on the PESI (and a number of further exclusion criteria), only one of 171 patients (0.6\%) developed recurrent venous thromboembolism within 90 days, indicating that outpatient care is safe and effective in place of inpatient care in selected low-risk PE patients [2]. At the other end of the risk spectrum, intermediate-high-risk patients (defined as RV dysfunction on TTE or CT and elevated troponin levels) benefit from thrombolytic therapy with tenecteplase as shown in the PEITHO study (OR 0.44, 95\% CI 0.230.87). This benefit, however, was counterbalanced by an increase in major, in particular intracranial, bleeding [6]. Likewise, in a recent meta-analysis, thrombolytic therapy was associated with a reduction of the combined end-point of death or treatment escalation and PE-related mortality at the cost of an increased risk of major haemorrhage and fatal or intracranial bleeding [5]. Based on this evidence and numerous available scores and models for risk stratification of not-high-risk PE developed and validated in cohort studies during the past years [7-9, 13, 15-19], a novel algorithm for risk assessment was introduced by the 2014 ESC guidelines [1]. In the present study focusing on 388 not-high-risk patients from a single-centre cohort, the new 2014 ESC algorithm was superior compared with the algorithm proposed by the 2008 ESC guidelines.

Given the relatively (compared with cohort studies) low complication rate of patients in the placebo group of the PEITHO study (28/499 (5.6\%) patients reached the primary end-point) and the insufficient predictive value of single markers such as imaging parameters or laboratory testing [17, 20, 21], Bova et al. [10] post hoc developed a multidimensional seven-point risk score based on four different weighted variables in 2874 patients from six cohort studies (table 1). Patients classified in stage III ( $>4$ points, intermediate-high-risk) had a $29.2 \%$ PE-related complication rate which was confirmed in the validation study of 1358 patients (42.0\% 30-day PE-related complications) [11]. In our present study, 19.0\% of the patients with a Bova score $>4$ points had an adverse 30 -day outcome and $6.3 \%$ died due to PE.

Another approach to identify intermediate-high-risk PE patients is the FAST score (table 1) [8, 9]. hsTnT has been demonstrated to improve risk stratification of PE patients compared with a "conventional" cardiac troponin $\mathrm{T}$ assay [13], and reliably identifies low-risk [13, 15] and intermediate-risk patients if an age-adjusted cut-off value ( $\geqslant 45 \mathrm{pg} \cdot \mathrm{mL}^{-1}$ for patients aged $\geqslant 75$ years) is used [14]. In the present study we could demonstrate that replacement of H-FABP by hsTnT using an age-adjusted cut-off value (modified FAST score) provides good prognostic performance (OR 15.9, 95\% 5.3-47.6, p<0.001; AUC 0.82, 95\% CI 0.75-0.86) which was comparable to the findings of the derivation (OR 11.6, 95\% 2.4-56.2, $\mathrm{p}=0.002$ [8]) and validation cohort (OR 18.0, 95\% CI 5.1-63.7, $\mathrm{p}<0.001$ [9]) of the original FAST score. Thus, pending external validation, the inclusion of a biomarker that is (in contrast to H-FABP) available in the majority of hospitals as part of routine laboratory diagnostic in the modified FAST score may represent a valuable alternative for rapid risk stratification of normotensive PE patients without the need for imaging tests.

Comparing these risk assessment strategies in 388 normotensive patients with acute PE, the findings of our present study can be summarised as follows: 1) regardless of the score or algorithm used, the rate of 
an adverse 30-day outcome was highest in the intermediate-high-risk classes; 2) all patients classified in low-risk classes had a favourable 30 -day outcome (0-1.4\% adverse outcome) and 3) none of these patients died of PE during the first 30-days; 4) the algorithm proposed by the 2014 ESC guidelines reached a higher AUC with regard to an adverse 30-day outcome compared with the algorithm proposed by the 2008 ESC guidelines; 5) the highest odds ratio with regard to an adverse 30-day outcome was observed for the modified FAST score (OR 15.9, 95\% CI 5.3-47.6, p<0.001), indicating that this score may be more suitable for identification of intermediate-high-risk patients.

It needs to be mentioned that, in the present study, complete results of imaging procedures for RV assessment as well as hsTnT testing were available for all patients (regardless of their sPESI). This explains why the proportion of our patients classified as "low-risk" (defined by sPESI of 0 plus a normal RV on imaging plus a negative hsTnT test) was $16.2 \%$ and thus lower than that in previous cohort studies which defined "low-risk" based on a sPESI of 0 alone. Our strategy may be considered as deviating from the risk stratification algorithm proposed in the 2014 ESC guidelines. However, rather than contradicting our approach, these guidelines mention that "Patients in the PESI class I-II, or with sPESI of 0 , and elevated cardiac biomarkers or signs of RV dysfunction on imaging tests, are also to be classified into the intermediate-low-risk category. This might apply to situations in which imaging or biomarker results become available before calculation of the clinical severity index" [1]. As shown in online supplementary table S1, $34.5 \%$ of our patients would have been classified as "low-risk" based on a sPESI of 0 alone (without considering the imaging or troponin results in that group) with one of them (0.7\%) having an adverse 30-day outcome. Importantly, the AUC of the dichotomised ESC algorithm would remain almost identical to that shown in table 3 as the proportion of patients in the intermediate-high-risk category would not be affected.

Some potential limitations of our study also need to be mentioned. First, for calculation of the sPESI, missing variables were considered to be normal. However, since data were prospectively collected, missing values could have affected classification in only $4.5 \%$ of all patients, and almost all variables required for calculation of the Bova score and the modified FAST score as well as for the algorithms proposed by the 2008 and 2014 ESC guidelines were available. Although only $61.6 \%$ of all patients underwent TTE, RV function could be assessed in $97.7 \%$ of all patients when information obtained from (diagnostic) CT was also taken into consideration. Second, although the number of patients with an adverse 30-day outcome $(n=25)$ was low given the single-centre study design, the rate of an adverse 30-day outcome (6.4\%) was comparable to other registries, cohort studies and trials $[6,10,15,17,22,23]$. Finally, the inclusion period (September 2007 to August 2014) partly overlapped with those of the derivation (October 2005 to April 2009 [8]) and validation (October 2005 to August 2011 [9]) study of the original FAST score based on the same single-centre prospective cohort (PERGO). However, the present study included 178 patients $(45.9 \%)$ who had neither been described in the derivation nor in the validation study of the original FAST score. Additionally, the present study is the first to investigate a modified version of the FAST score using hsTnT instead of H-FABP.

In conclusion, good prognostic performance was observed for the modified FAST score if an age-adjusted hsTnT cut-off value was used for calculation instead of H-FABP. The algorithm proposed by the 2014 ESC guidelines and the modified FAST score and Bova score appear more suitable for risk stratification of normotensive PE patients compared with the algorithm proposed by the previous 2008 ESC guidelines. All algorithms and scores safely identify low-risk patients, while the modified FAST score appears more suitable to identify intermediate-high-risk patients. Depending on external validation of our findings, the modified FAST score might improve and simplify risk stratification of normotensive PE patients.

\section{Acknowledgements}

We thank Valerie Seeber (Center for Thrombosis and Hemostasis, University Medical Center Mainz, Germany) for statistical assistance and advice. We thank Johanna Schwung and Caroline Niemann (Clinic of Cardiology and Pneumology, Heart Center, University of Göttingen, Germany) for assistance in collecting the clinical data of the study patients. This publication is part of the medical doctoral thesis of L. Hobohm.

\section{References}

1 Konstantinides SV, Torbicki A, Agnelli G, et al. 2014 ESC Guidelines on the diagnosis and management of acute pulmonary embolism. Eur Heart J 2014; 35: 3033-3080.

2 Aujesky D, Roy PM, Verschuren F, et al. Outpatient versus inpatient treatment for patients with acute pulmonary embolism: an international, open-label, randomised, non-inferiority trial. Lancet 2011; 378: 41-48.

3 Zondag W, Mos IC, Creemers-Schild D, et al. Outpatient treatment in patients with acute pulmonary embolism: the Hestia Study. J Thromb Haemost 2011; 9: 1500-1507.

4 Lankeit M, Konstantinides S. Is it time for home treatment of pulmonary embolism? Eur Respir J 2012; 40: 742-749.

5 Marti C, John G, Konstantinides S, et al. Systemic thrombolytic therapy for acute pulmonary embolism: a systematic review and meta-analysis. Eur Heart J 2015; 36: 605-614.

6 Meyer G, Vicaut E, Danays T, et al. Fibrinolysis for patients with intermediate-risk pulmonary embolism. $N$ Engl J Med 2014; 370: 1402-1411. 
Jiménez D, Aujesky D, Moores L, et al. Simplification of the Pulmonary Embolism Severity Index for prognostication in patients with acute symptomatic pulmonary embolism. Arch Intern Med 2010; 170: 1383-1389.

8 Lankeit M, Friesen D, Schäfer K, et al. A simple score for rapid risk assessment of non-high-risk pulmonary embolism. Clin Res Cardiol 2013; 102: 73-80.

9 Dellas C, Tschepe M, Seeber V, et al. A novel H-FABP assay and a fast prognostic score for risk assessment of normotensive pulmonary embolism. Thromb Haemost 2014; 111: 996-1003.

10 Bova C, Sanchez O, Prandoni P, et al. Identification of intermediate-risk patients with acute symptomatic pulmonary embolism. Eur Respir J 2014; 44: 694-703.

11 Fernández C, Bova C, Sanchez O, et al. Validation of a model for identification of patients at intermediate to high risk for complications associated with acute symptomatic pulmonary embolism. Chest 2015; 148: 211-218.

12 Torbicki A, Perrier A, Konstantinides S, et al. Guidelines on the diagnosis and management of acute pulmonary embolism. The Task Force for the Diagnosis and Management of Acute Pulmonary Embolism of the European Society of Cardiology (ESC). Eur Heart J 2008; 29: 2276-2315.

13 Lankeit M, Friesen D, Aschoff J, et al. Highly sensitive troponin T assay in normotensive patients with acute pulmonary embolism. Eur Heart J 2010; 31: 1836-1844.

14 Kaeberich A, Seeber V, Jiménez D, et al. Age-adjusted high-sensitivity troponin T cut-off value for risk stratification of pulmonary embolism. Eur Respir J 2015; 45: 1323-1331.

15 Lankeit M, Jiménez D, Kostrubiec M, et al. Predictive value of the high-sensitivity troponin $\mathrm{T}$ assay and the simplified Pulmonary Embolism Severity Index in hemodynamically stable patients with acute pulmonary embolism: a prospective validation study. Circulation 2011; 124: 2716-2724.

16 Lankeit M, Jiménez D, Kostrubiec M, et al. Validation of N-terminal pro-brain natriuretic peptide cut-off values for risk stratification of pulmonary embolism. Eur Respir J 2014; 43: 1669-1677.

17 Jiménez D, Aujesky D, Moores L, et al. Combinations of prognostic tools for identification of high-risk normotensive patients with acute symptomatic pulmonary embolism. Thorax 2011; 66: 75-81.

18 Klok FA, Van Der Bijl N, Eikenboom HC, et al. Comparison of CT assessed right ventricular size and cardiac biomarkers for predicting short-term clinical outcome in normotensive patients suspected of having acute pulmonary embolism. J Thromb Haemost 2010; 8: 853-856.

19 Sanchez O, Trinquart L, Caille V, et al. Prognostic factors for pulmonary embolism: the PREP study, a prospective multicenter cohort study. Am J Respir Crit Care Med 2010; 181: 168-173.

20 Becattini C, Casazza F, Forgione C, et al. Acute pulmonary embolism: external validation of an integrated risk stratification model. Chest 2013; 144: 1539-1545.

21 Klok FA, Meyer G, Konstantinides S. Management of intermediate risk pulmonary embolism: uncertainties and challenges. Eur J Haematol 2015; 95: 489-497.

22 Aujesky D, Stone RA, Kim S, et al. Length of hospital stay and postdischarge mortality in patients with pulmonary embolism. Arch Intern Med 2008; 168: 706-712.

23 Pollack CV, Schreiber D, Goldhaber SZ, et al. Clinical characteristics, management, and outcomes of patients diagnosed with acute pulmonary embolism in the emergency department initial report of EMPEROR (Multicenter Emergency Medicine Pulmonary Embolism in the Real World Registry). J Am Coll Cardiol 2011; 57: 700-706. 